\author{
Andreas Gass \\ Josef Flammer \\ Lilly Linder \\ Silvana C. Romerio \\ Paul Gasser \\ Walter E. Haefeli
}

\section{Inverse correlation between endothelin-1- induced peripheral microvascular vasoconstriction and blood pressure in glaucoma patients}

Received: 19 August 1996

Revised version received: 4 March 1997

Accepted: 2 April 1997
A. Gass - J. Flammer - S.C. Romerio

P. Gasser

Department of Ophthalmology,

University of Basel, Switzerland

A. Gass - L. Linder - S.C. Romerio

W.E. Haefeli (

Division of Clinical Pharmacology,

Department of Medicine,

University Hospital,

CH-4031 Basel, Switzerland,

Tel. +41-61-265 25 25;

Fax +41-61-265 45 60;

e-mail haefeli@ubaclu.unibas.ch

L. Linder - W.E. Haefeli

Department of Pharmacy,

University of Basel, Switzerland

P. Gasser

Medical Outpatient Department,

University Hospital, CH-4031 Basel,

Switzerland
Abstract - Background: The potent vasoconstrictor peptide endothelin- 1 has been shown to participate in the control of peripheral vascular tone and in the regulation of ocular perfusion. In glaucoma patients vasospasms and arterial hypotension have been identified as risk factors for the progression of glaucomatous damage, and the regulation of endothelin-1 release is disturbed in some of these patients. The aim of this study was to assess the relationship between resting blood pressure and cutaneous vascular responsiveness to endothelin1 and phenylephrine in patients with glaucoma and in matched controls. Methods: In 9 patients with primary open-angle glaucoma (POAG), 7 patients with normal tension glaucoma (NTG), and 16 age- and sex-matched controls, endothelin-1 and phenylephrine responses were assessed in the human forearm microcirculation using laser Doppler flowmetry during intra-arterial drug administration. Blood pressure was measured intraarterially. Results: In contrast to $\alpha_{1}$-adrenergic effects, endothelin-1 responses were inversely correlated to both systolic $\left(r^{2}=0.27\right.$, $P=0.05)$ and diastolic $\left(r^{2}=0.54\right.$, $P=0.001$ ) blood pressure in glaucoma patients, whereas there was no such correlation in controls. $\mathrm{Pa}$ tients with lower blood pressure values were more sensitive to the vasoconstrictor effects of endothelin-1. Cutaneous responsiveness to endothelin-1 and phenylephrine was similar in glaucoma patients and in controls. Conclusion: These results reveal that glaucoma patients appear to have peripheral microvascular abnormalities which are exhibited as altered responsiveness to endothelin-1. Thus, this study supports the hypothesis that endothelin-1-related microvascular dysfunction may be involved in the pathogenesis of glaucomatous damage.

\section{Introduction}

Glaucomatous damage of the eye is characterized by progressive nerve fiber loss combined with excavation of the optic nerve head and visual field damage. Besides increased intraocular pressure and systemic arterial hypotension $[15,17]$, vasospastic diathesis has been identified as a further risk factor for the occurrence and progression of such a damage $[8,12]$. Hence, in the pathogenesis of glaucoma additional factors affecting the vas- culature in general must be involved [9]. Also circulatory defects of the optic disk have been described in patients with glaucoma [32], although it is still unclear whether these disturbances are cause or consequence of the visual field defects. One of the major endogenous factors regulating eye perfusion is endothelin-1 [23], the plasma concentration of which is increased in those glaucoma patients who have normal intraocular pressure [18, 24]. In the present study we evaluated the sensitivity of the microcirculation to two prototype vasoconstrictors, 
phenylephrine and endothelin-1, and assessed the relationship between individual vascular responsiveness and resting intra-arterial blood pressure in glaucoma patients and in healthy controls.

\section{Patients and methods}

After giving written informed consent, 16 age- and sex-matched controls (mean age 63 years, range $28-73 ; 7$ women, 9 men) and 16 otherwise healthy glaucoma patients (mean age: 61 years, range $26-74 ; 6$ women, 10 men) with open anterior chamber angle and characteristic glaucomatous visual field defects and/or optic nerve damage were included. The study was approved by the ethics committee of the Department of Medicine, University Hospital Basel, Switzerland. Nine patients had primary open-angle glaucoma (POAG; intraocular pressure consistently $>21 \mathrm{mmHg}$; mean age \pm SEM $62 \pm 4$ years, range 34-71 years), and seven had normal-tension glaucoma (NTG; untreated intraocular pressure $\leq 21 \mathrm{mmHg}$; age $59 \pm 6$ years, range $26-75$ years). Patients and controls underwent complete blood analysis for exclusion of severe hematologic, renal, or hepatic dysfunction. In addition, serum lipids, including HDL and LDL fractions, were determined and were found to be similar in both groups. Patients with known vascular disease, concurrent drug use, or smoking were excluded. In glaucoma patients, systemically administered drugs were discontinued at least 14 days, topically administered drugs at least 7 days before the study. Controls were not taking any medication, were not suffering from any concurrent disease, and their medical history was not remarkable for cardiovascular, liver or kidney disorders.

All studies were done in the morning with the patient semirecumbent in bed. Under local anesthesia an 18-gauge catheter was inserted into the left brachial artery for blood pressure recording (Statham $\mathrm{P} 23 \mathrm{~Pb}$ pressure transducer) and regional drug administration. Drugs were infused at a constant flow rate $(1 \mathrm{ml} / \mathrm{min})$, and doses were adjusted according to forearm volume. After reaching a stable baseline during infusion of solvent (Physiogel, $4 \%$ gelatin solution, Swiss Red Cross, Bern, Switzerland), increasing doses of phenylephrine (Neo-Synephrine, Winthrop, Münchenstein, Switzerland; $0.3-10 \mu \mathrm{g} / \mathrm{min} / 100 \mathrm{ml}$ tissue) and endothelin-1 (Clinalfa AG, Läufelfingen, Switzerland; $0.005-25 \mathrm{ng} / \mathrm{min} /$ $100 \mathrm{ml}$ tissue) were administered into the brachial artery for $5 \mathrm{~min}$ to construct cumulative dose-response curves. The two drug administrations were separated by a washout phase of sufficient length to allow forearm blood flow to return to baseline values (approximately $60 \mathrm{~min}$ ). The short-acting phenylephrine was always administered first, since the effect of endothelin-1 may last for several hours and may, therefore, confound the effects of subsequently administered vasoactive compounds [37]. Changes in cutaneous blood cell velocity were measured with a laser Doppler flowmeter [2-mW helium-neon laser, light wavelength $632.8 \mathrm{~nm}$, wide band mode (20-12000 Hz); Periflux PF3, Perimed, Sweden] [3]. The laser probe (PF 308, Periflux) was fixed on the lateral ventral forearm with a thermostat probe holder (PF 206, Periflux) heated to $34^{\circ} \mathrm{C}$. The baseline immediately before drug application was set to $100 \%$, and flow was expressed as percentage change from this baseline. The last $24 \mathrm{~s}$ of each drug infusion was used for evaluation.

Individual phenylephrine dose-response curves, expressed as percentage change from baseline, were fitted to a sigmoid model by use of a nonlinear least-squares regression program (Allfit) providing estimates of the maximum effect (Emax) and the doserate producing $50 \%$ of Emax $\left(\mathrm{ED}_{50}\right)$ [6]. Endothelin-1 dose-response curves were biphasic and, therefore, not evaluated with a sigmoid model. For these semilogarithmic curves, area under the curve (AUC) values were calculated using the trapezoidal rule, with vasodilation expressed as positive and constriction as nega- tive values. Group comparisons were done with the Mann-Whitney $U$-test, and correlations were done by linear regression analysis. Pairs of averaged dose-response curves were evaluated with analysis of variance (ANOVA) for repeated measurements. A $P$ value of $<0.05$ was considered significant.

\section{Results}

Intra-arterial systolic and diastolic blood pressure values measured at baseline before administration of drugs were $117 \pm 4 / 56 \pm 1 \mathrm{mmHg}$ in glaucoma patients and $122 \pm 3 / 57 \pm 2 \mathrm{mmHg}$ in the control group $(P>0.3$ for diastolic and systolic values). The effect of intra-arterial endothelin-1 was biphasic in all controls and in all but one patient with POAG (Fig. 1), and the dose-response

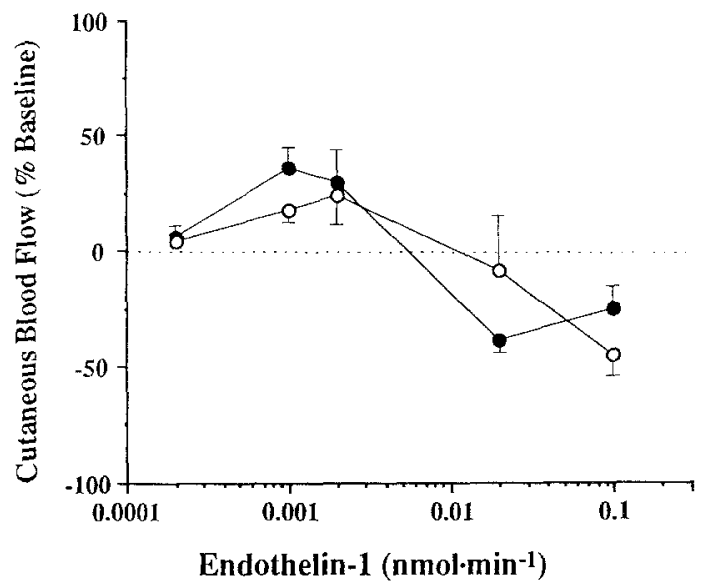

Fig. 1 Mean changes in cutaneous blood flow recorded in 16 glaucoma patients $(O)$ and in 16 age- and sex-matched controls $(O)$ during intra-arterial administration of increasing dosages of endothelin-1

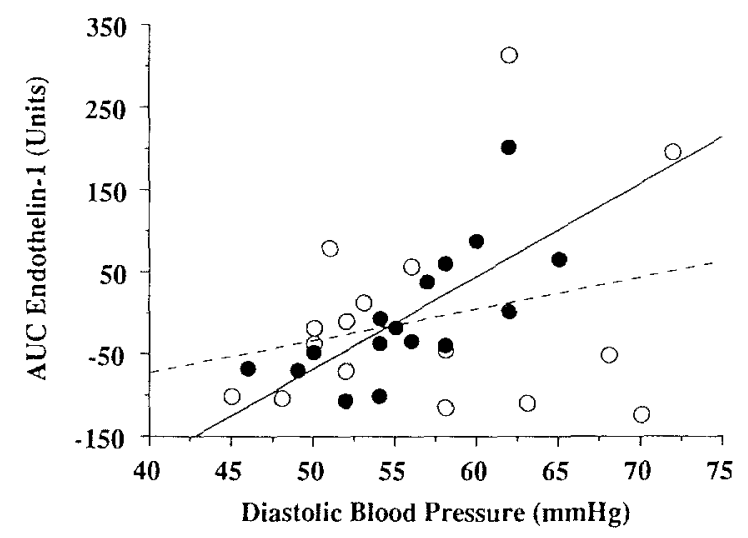

Fig. 2 Linear correlation between cutaneous vasoconstriction after infusion of endothelin-1 (expressed as AUC values) and intraarterial diastolic blood pressure $(\mathrm{mmHg})$ at baseline in 16 patients with glaucoma $(\mathbf{O}) ; r^{2}=0.52, P=0.002 ; \mathrm{AUC}=-634+11.3$ (-) (diastolic blood pressure)] and 16 age- and sex-matched controls $\left(O ; r^{2}=0.068, P=0.33\right)$ 


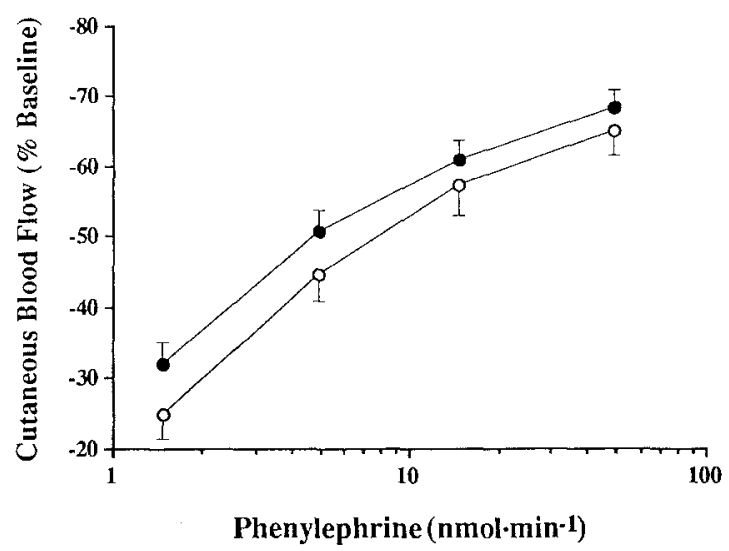

Fig. 3 Mean changes in cutaneous blood flow recorded in 16 patients with glaucoma (O) and 16 age- and sex-matched controls (O) during intra-arterial administration of increasing dosages of phenylephrine. Emax and $\mathrm{ED}_{50}$ values of the two fitted doseresponse curves were not significantly different

curves of the two groups were similar $(P=0.8)$. At lower dose-rate the peptide induced vasodilation, whereas at higher dose-rate pronounced vasoconstriction occurred. While there was no significant relationship between blood pressure values and endothelin-1 responsiveness in control subjects, vasoconstriction was inversely correlated with intra-arterial blood pressure in glaucoma patients. Evaluation by linear regression revealed $r^{2}$ values of $0.3(P=0.03)$ for systolic, $0.39(P=0.007)$ for mean, and $0.47(P=0.003)$ for diastolic blood pressure. Also when expressed as AUC values, dose-response relationships of endothelin-1 were correlated with systolic $\left(r^{2}=0.27, P=0.05\right)$, mean $\left(r^{2}=0.5, P=0.003\right)$, and diastolic blood pressure $\left(r^{2}=0.54, P=0.001\right)$ (Fig. 2).

The mean phenylephrine dose-response relationships of the two groups were similar $(P=0.3)$ and are shown in Fig. 3. Fitted mean $\log \mathrm{ED}_{50}(\log \mathrm{ng} / \mathrm{min}, P=0.37)$ and Emax (vasoconstriction expressed as percentage of baseline, $P=0.49$ ) values for both groups were similar (controls: $-0.35 \pm 0.1,65 \% \pm 3.6$; glaucoma: $0.47 \pm 0.1,70 \% \pm 2.9$ ). There was no correlation between $\alpha_{1}$-adrenergic sensitivity (expressed as $\log E D_{50}$ ) or efficacy (expressed as Emax) and intra-arterial systolic or diastolic blood pressure, either in controls or in glaucoma patients.

\section{Discussion}

The aim of this investigation was to test the main vasoconstrictor pathways involved in the regulation of vascular tone in the human peripheral microcirculation. Although the regulation of the ocular circulation might be different from peripheral vascular beds [15], there is evidence for a relationship between peripheral forearm circulation and the ocular circulation in patients with va- sospastic syndromes [13]. The forearm vasculature was also chosen because this arterial bed only rarely develops arteriosclerosis, and changes in vascular responsiveness therefore reflect functional rather than anatomic alterations of the vessels. Moreover, this vascular bed appeared particularly suitable for these experiments since it has repeatedly been used to characterize functional abnormalities of vessels in patients with diseases affecting the vasculature $[5,16,21,27]$ and to study responsiveness of the skin microcirculation [3,35]. Finally, since only small doses are required to construct complete dose-response curves, systemic counter-regulatory reflex activation is unlikely to occur or to confound the results.

Whereas $\alpha_{1}$-adrenoceptor-mediated vasoconstriction was independent of blood pressure, endothelin-1 responses were inversely correlated with systolic and diastolic values in glaucoma patients, indicating that patients with lower blood pressure were more sensitive to endothelin-1-induced vasoconstriction. This is of interest, since low blood pressure may promote glaucomatous damage although it is unclear by which mechanism. Approximately $50 \%$ of the correlation between endothelin1 responses and diastolic blood pressure in glaucoma patients was explained by this relationship. This is a rather remarkable finding, since vascular tone of forearm resistance arteries is also modulated by other effector pathways such as spontaneous nitric oxide release [34]. To our knowledge, no data about the sensitivity of human vessels to endothelin-1 with respect to blood pressure are available. However, in accordance with our findings, in hypertensive rats, the sensitivity of vascular smooth muscle to endothelin-1 is reduced [7]. In the same study the response to norepinephrine did not differ between normo- and hypertensive animals, indicating that the reduced sensitivity is endothelin-1-specific. Similar results have also been reported in small subcutaneous arteries from patients with essential hypertension [33].

In almost all patients studied, endothelin-1 evoked a biphasic response in cutaneous blood flow with vasodilation at lower and pronounced vasoconstriction at higher dose-rates. Biphasic effects of endothelin-1 have been reported earlier [20] and have been explained by the existence of at least two receptors $\left(\mathrm{ET}_{\mathrm{A}}\right.$ and $\left.\mathrm{ET}_{\mathrm{B}}\right)$. Whereas the endothelium primarily expresses vasodilator $\mathrm{ET}_{\mathrm{B}}$ receptors, vasoconstrictive $\mathrm{ET}_{\mathrm{A}}$ and $\mathrm{ET}_{\mathrm{B}}$ receptors are located on vascular smooth muscle cells [23]. Evidence has also been presented for the presence of both endothelin receptor subtypes in the microcirculation of porcine eyes and in isolated human choroidal and retinal vessels [22], indicating that endothelin-1 can evoke dose-dependent vasodilator and vasoconstrictor effects also in the ocular microcirculation.

In accordance with findings in patients with other vasospastic disorders (Raynaud's disease [1]), $\alpha_{1}$-adrenergic responsiveness in glaucoma patients was similar to 
controls, suggesting that differences in peripheral $\alpha_{1}$ adrenoceptor sensitivity are absent and do not promote generalized vasospasms which are known to occur in certain glaucoma patients.

The cause for the relationship between blood pressure and endothelin-1 responsiveness in the microcirculation of glaucoma patients is unknown, and the design of this study does not allow us to conclude whether these parameters are only associated with each other or whether any of them is causally involved in the pathogenesis of glaucoma. However, since endothelin-1 responses are functionally linked to the activity of nitric oxide synthases, and since chronically administered NO may increase both the expression of $\mathrm{ET}_{\mathrm{A}}$ receptors and the affinity of the peptide to its receptor [30], it may be speculated that the blunted constrictor responses to endothelin- 1 in hypertensive patients [33] and animals [7] are caused by decreases in NO production, which has been shown in some [21] but not all studies addressing this issue [27]. Collectively, these data may suggest that glaucoma patients tend to have higher NO production, which could subsequently cause increased vasoconstrictor responses to endothelin-1. Hence, future studies should focus on the evaluation of the effects of endothelin-1, its antagonists, and on the investigation of NO synthase activities in these patients.

Obviously the results of this study were obtained in the peripheral microcirculation of the human forearm and not in the ocular circulation; therefore, they can only be extrapolated to the eye circulation. The local regulation of the ocular circulation is complex, has not yet been studied in all details $[10,36]$, and may differ in critical aspects from the peripheral microcirculation. Whereas choroidal vessels are amply innervated $[11,25]$ and flow is largely independent of autoregulation [19], retinal vessels are not innervated and local flow is controlled by efficient autoregulatory mechanisms similar to those regulating cerebral blood flow $[2,29]$. The vascular area of the optic nerve head which is critical in the pathogenesis of glaucoma also lacks autonomic innervation and is to some extent autoregulated $[26,28,31]$. However, blood supply of the optic nerve head mainly depends on the perfusion of the posterior ciliary arteries, which are regulated in a way similar to coronary and peripheral arteries [14]. In particular, all these ocular and extraocular vessels have in common that they are rather sensitive to the vasoconstrictor effects of endothelin-1 [4, 22, 23]. Thus, since systemic blood pressure is linked to peripheral vascular sensitivity it appears possible that there is also a correlation with ocular vascular responsiveness in these patients. This, however, remains to be shown in future studies including the ocular circulation.

Acknowledgements We would like to thank Andreas J. Bircher, M.D. for technical support. These studies were supported by grant 32-36575.92 and 32-49825.96 from the Swiss National Research Foundation and by a grant from Sandoz Foundation, Basel, Switzerland.

\section{References}

1. Bedarida GV, Kim D, Blaschke TF, Hoffman BB (1993) Venodilation in Raynaud's disease. Lancet 342: 14511454

2. Bill A, Nilsson SFE (1985) Control of ocular blood flow. J Cardiovasc Pharmacol 7 [suppl 3]:S96-S102

3. Bircher A, De Boer EM, Agner T, Wahlberg JE, Serup J (1994) Guidelines for measurement of cutaneous blood flow by laser Doppler flowmetry. Contact Dermatitis 30: 65-72

4. Bursell SE, Clermont AC, Oren B, King GL (1995) The in vivo effect of endothelins on retinal circulation in nondiabetic and diabetic rats. Invest Ophthalmol Vis Sci 36:596-607

5. Chowienczyk PJ, Watts GF, Cockcroft JR, Ritter JM (1992) Impaired endothelium-dependent vasodilation of forearm resistance vessels in hypercholesterolemia. Lancet 340: 14301432
6. De Lean A, Munson PJ, Rodbard D (1978) Simultaneous analysis of families of sigmoidal curves: application to bioassay, radioligand assay, and physiological dose-response curves. Am J Physiol 235:E97-E102

7. Dohi Y, Lüscher TF (1991) EndotheIin in hypertensive resistance arteries. Intraluminal and extraluminal dysfunction. Hypertension 18:543-549

8. Drance SM, Douglas GR, Wijsman K, Schulzer M, Britton RJ (1988) Response of blood flow to warm and cold in normal and low-tension glaucoma patients. Am J Ophthalmol 105:35-39

9. Flammer J (1995) To what extent are vascular factors involved in the pathogenesis of glaucoma? In: Kaiser HJ, Flammer J, Hendrickson P (eds) Ocular blood flow. Glaucoma Meeting. Karger, Basel, pp 12-39

10. Flammer J, Orgül S (1997) Optic nerve blood flow abnormalities in glaucoma. Prog Retin Res (in press)

11. Flügel-Koch C, Kaufman P, LütjenDrecoll E (1994) Association of a choroidal ganglion cell plexus with the fovea centralis. Invest Ophthalmol $V$ is Sci 35:4268-4272
12. Gasser P, Flammer J (1991) Bloodcell velocity in the nailfold capillaries of patients with normal-tension and high-tension glaucoma. Am J Ophthalmol 111:585-588

13. Guthauser U, Flammer J, Mahler F (1988) The relationship between digital and ocular vasospasm. Graefe's Arch Clin Exp Ophthalmol 226:224226

14. Haefliger IO, Meyer P, Flammer J, Luischer TF (1994) The vascular endothelium as a regulator of the ocular circulation: a new concept in ophthalmology? Surv Ophthalmol 39: $123-$ 132

15. Hayreh SS (1995) The optic nerve head circulation in health and disease. Exp Eye Res 61:259-272

16. Indolfi $\mathrm{C}$, Maione $\mathrm{AG}$, Volpe $\mathrm{M}, \mathrm{Ra}-$ pacciuolo A, Esposito G, Ceravolo R, Rendina V, Condorelli M, Chiariello M (1994) Forearm vascular responsiveness to $\alpha_{1}$ - and $\alpha_{2}$-adrenoceptor stimulation in patients with congestive heart failure. Circulation 90: 17-22 
17. Kaiser HJ, Flammer J, Graf T, Stümpfig D (1993) Systemic blood pressure in glaucoma patients. Graefe's Arch Clin Exp Ophthalmol 231: 677-680

18. Kaiser HJ, Flammer J, Wenk M, Lüscher TF (1995) Endothelin-1 plasma levels in normal-tension glaucoma: abnormal response to postural changes. Graefe's Arch Clin Exp Ophthalmol 233: 484-488

19. Kiel JW (1995) The effect of arterial pressure on the ocular pressure-volume relationship in the rabbit. Exp Eye Res 60: 267-278

20. Kiowski W, Linder L (1992) Reversal of endothelin-1-induced vasoconstriction by nifedipine in human resistance vessels in vivo in healthy subjects. Am J Cardiol 69: 1063-1066

21. Linder L, Kiowski W, Bühler FR, Liischer TF (1990) Indirect evidence for release of endothelium-derived relaxing factor in human forearm circulation in vivo. Blunted response in essential hypertension. Circulation 81: 1762-1767

22. MacCumber MW, D'Anna SA (1994) Endothelin receptor-binding subtypes in the human retina and choroid. Arch Ophthalmol 112: 1231-1235
23. Meyer P, Flammer J, Lüscher TF (1993) Endothelium-dependent regulation of the ophthalmic microcirculation in the perfused porcine eye: role of nitric oxide and endothelins. Invest Ophthalmol Vis Sci 34:3614-3621

24. Moriya S, Sugiyama T, Shimizu K, Hamada J, Tokuoka S, Azuma I (1992) Low-tension glaucoma and endothelin (ET-1). Folia Ophthalmol Jpn 43:554-559

25. Nakanome Y, Karita K, Izumi H, Tamai M (1995) Two types of vasodilation in cat choroid elicited by electrical stimulation of the short ciliary nerve. Exp Eye Res 60:37-42

26. Orgül S, Meyer P, Cioffi GA (1995) Physiology of blood flow regulation and mechanisms involved in optic nerve perfusion. J Glaucoma 4:427443

27. Panza JA, Quyyumi AA, Brush JE, Epstein SE (1990) Normal endothelium-dependent vascular relaxation in patients with essential hypertension. N Engl J Med 323:22-27

28. Pillunat LE, Anderson DR, Knighton RW, Joos KM, Feuer WJ (1996) Autoregulation of ocular blood flow. In: Kaiser HJ, Flammer J, Hendrickson P (eds) Ocular blood flow. Glaucoma Meeting. Karger, Basel, pp 138-144

29. Pournaras CJ (1996) Autoregulation of ocular blood flow. In: Kaiser HJ, Flammer J, Hendrickson P (eds) Ocular blood flow. Glaucoma Meeting. Karger, Basel, pp 40-50

30. Redmond EM, Cahill PA, Hodges R, Zhang S, Sitzmann JV (1996) Regulation of endothelin receptors by nitric oxide in cultured rat vascular smooth muscle cells. J Cell Biol 166:469-479
31. Riva CE, Harino S, Petrig BL, Shonat RD (1992) Laser Doppler flowmetry in the optic nerve. Exp Eye Res 55: 499-506

32. Schwartz B (1994) Circulatory defects of the optic disk and retina in ocular hypertension and high pressure open-angle glaucoma. Surv Ophthalmol 38 [Supp1]:S23-S34

33. Schiffrin EL, Deng LY, Larochelle P (1992) Blunted effects of endothelin upon small subcutaneous resistance arteries of mild essential hypertensive patients. J Hypertension 10:437-444

34. Vallance P, Collier J, Moncada S (1989) Effects of endothelium-derived nitric oxide on peripheral arteriolar tone in man. Lancet ii:997-1000

35. Wenzel RR, Noll G, Lüscher TF (1994) Endothelin receptor antagonists inhibit endothelin in human skin microcirculation. Hypertension 23:581-586

36. Williamson TH, Harris A (1994) Ocular blood flow measurement. Br J Ophthalmol 78:939-945

37. Yang Z, Richard V, von Segesser L, Bauer E, Stulz P, Turina M, Lüscher TF (1990) Threshold concentrations of endothelin-1 potentiate contractions to norepinephrine and serotonin in human arteries. A new mechanism of vasospasm? Circulation 82:188195 